Document downloaded from:

http://hdl.handle.net/10251/40670

This paper must be cited as:

González Martínez, AJ.; Peiró, A.; Conde, P.; Hernández Hernández, L.; Moliner Martínez, L.; Orero Palomares, A.; Rodríguez-Álvarez, M.... (2013). Monolithic crystals for PET devices: optical coupling optimization. Nuclear Instruments and Methods in Physics Research Section A: Accelerators, Spectrometers, Detectors and Associated Equipment. 731:288-294. doi:10.1016/j.nima.2013.05.049.

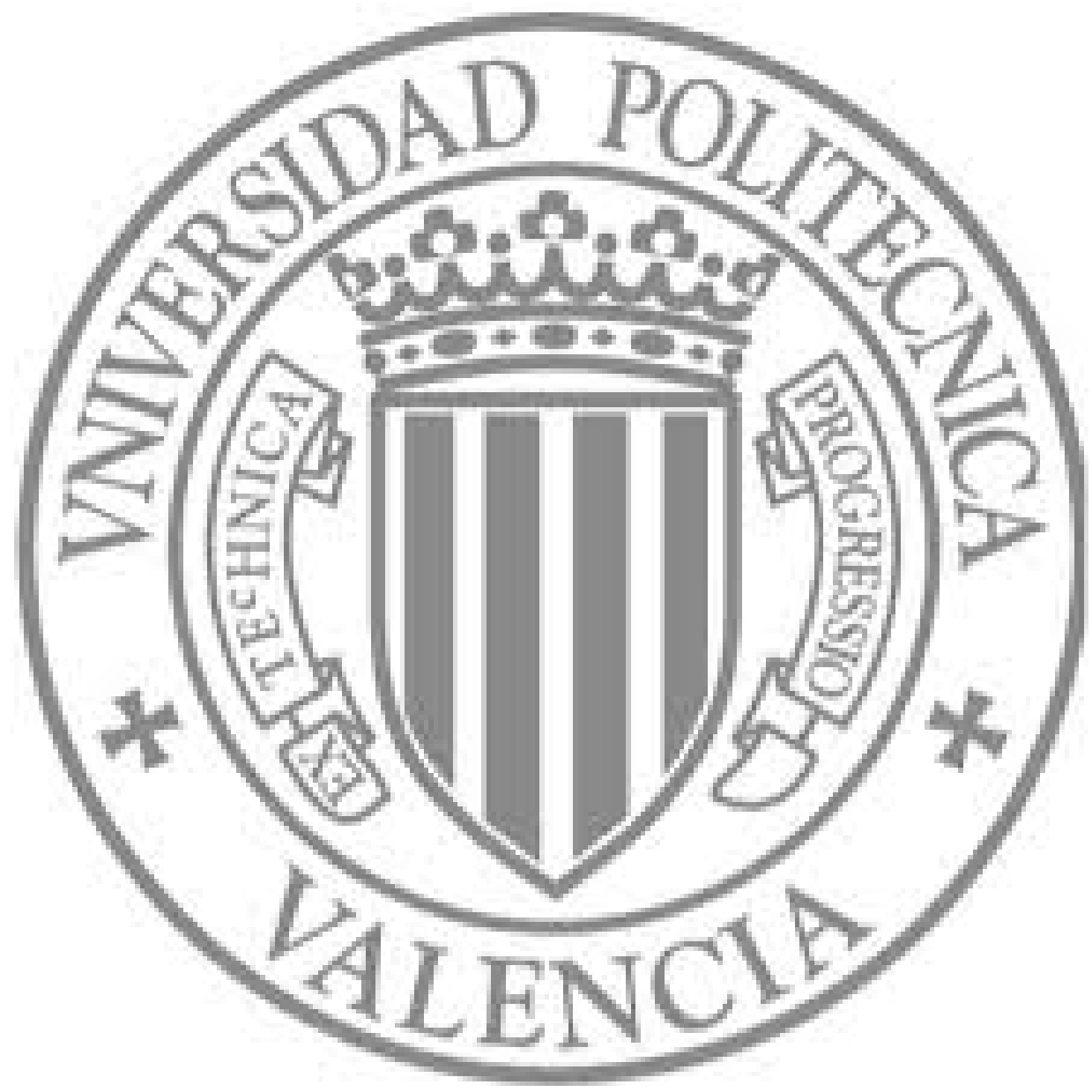

The final publication is available at

http://dx.doi.org/10.1016/j.nima.2013.05.049

Copyright Elsevier 
$3 \quad$ A.J. González ${ }^{\mathrm{a}, *}$, A. Peiróa ${ }^{2}$, P. Conde ${ }^{\mathrm{a}}$, L. Hernández $^{\mathrm{a}}, \mathrm{L}_{\text {L. Moliner }}^{\mathrm{a}}$, A.

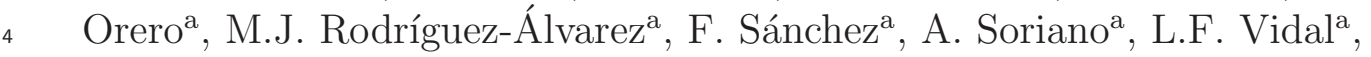

\section{Monolithic crystals for PET devices: optical coupling optimization}

J.M. Benlloch ${ }^{\mathrm{a}}$

${ }^{a}$ Institute for Instrumentation in Molecular Imaging (I3M). Centro Mixto UPV - CSIC

- CIEMAT. Camino de Vera s/n, 46022, Valencia, Spain.

\section{Abstract}

In this work we present a method to efficiently collect scintillation light when using monolithic scintillator crystals. The acceptance angle of the scintillation light has been reduced by means of optical devices reducing the border effect which typically affects continuous crystals. We have applied this procedure on gamma detectors for PET systems using both position sensitive PMTs and arrays of SiPMs. In the case of using SiPMs, this approach also helps to reduce the photosensor active area.

We evaluated the method using PMTs with a variety of different crystals with thicknesses ranging from 10 to $24 \mathrm{~mm}$. We found that our design allows the use of crystal blocks with a thickness of up to $18 \mathrm{~mm}$ without degrading the spatial resolution caused by edge effects and without a significant detriment to the energy resolution. These results were compared with simulated data. The first results of monolithic LYSO crystals coupled to an array of $256 \mathrm{SiPMs}$ by means of individual optical light guides are also presented.

\section{Keywords:}

Monolithic crystals, PSPMTs, SiPMs, Acceptance Angle reduction, 


\section{Introduction}

Most detector blocks used for $\gamma$-ray detection, especially for Positron Emission Tomography (PET), are primarily based on scintillation crystals and photosensors. The incoming radiation is commonly converted into scintillation light in order to be photodetected. There are basically two types of crystal configurations used for this purpose, namely pixelated and monolithic. Most commercial and academical PET systems are based on pixelated crystal designs. Regarding the photosensor technology, the options might also be divided in two groups. Most installed systems use Photomultiplier Tubes (PMTs) whereas in recent years, designs based on solid state technology are widely spread even in commercially available human devices ${ }^{(1,2)}$.

From the point of view of crystal type selection, a pixelated configuration allows an accurate photon impact determination for the planar impact position. Their resulting position accuracy strongly depends on the size of the individual pixel. In most of these approaches, every pixel is carefully treated. Their lateral and entrance faces are individually polished and covered with high efficiency reflectors in order to collect as much light as possible. Alternatively to pixelated assemblies, the use of continuous scintillation crystals permits preservation of the spatial distribution of the scintillation light generated for each $\gamma$-ray event ${ }^{(3-5)}$. The light distribution can be reconstructed with a small number of statistical moments allowing for accurate planar, as well as also depth of interaction (DOI), photon impact information ${ }^{(6)}$. This method permits simplification of the design of PET detectors in regards to the DOI information. However, such continuous crystals account for moder- 
ate edge effects which significantly depend on the crystal thickness. We have previously showed that several configurations for crystal surface treatment (for instance black paint) can preserve the original scintillation light distribution $^{(6)}$. Nevertheless, for thick crystals these methods are not sufficient for an accurate determination of the photon impact within the crystal volume due to the strong border effect ${ }^{(7)}$.

A reduction of the acceptance angle (AA) of the scintillation photons would minimize edge effects when working with monolithic crystals. However, a compromise with the amount of the transmitted light should be kept. Optical devices interfacing the scintillation crystal with the photosensor, such as the so-called faceplates (FPs) make possible to reduce the AA of the incoming $\operatorname{light}^{(7)}$.
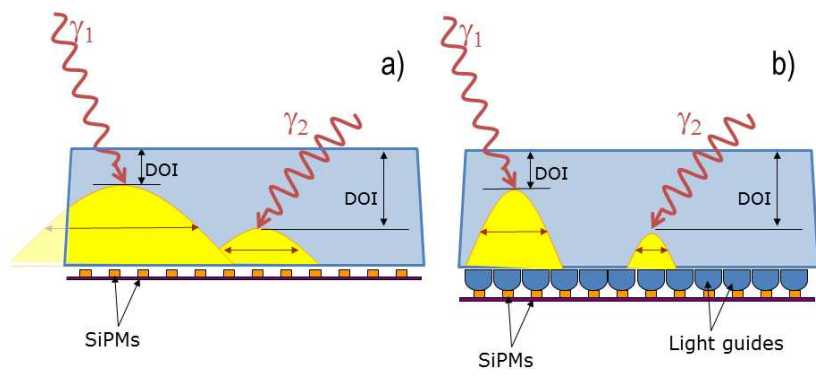

b)

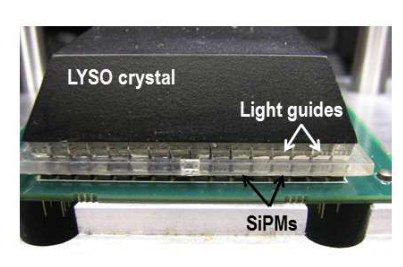

Figure 1: Example of the border effect present in detector blocks using a monolithic crystal design. (a) Sketch of direct coupling between the crystal and an array of SiPMs. The shadowed image represents the part of the distribution that would be absorbed, worsening the impact position determination. (b) Sketch of a crystal and SiPMs array using light guides as interface material. The use of certain light guides can reduce the acceptance angle of the scintillation light, schematically depicted in this figure by reducing the cone light angles. (c) Photograph of a crystal, light guides and SiPMs set-up. 
62

in the proportional (APDs) or in the Geiger regimes most commonly named as Silicon Photomultipliers (SiPMs). APDs and SiPMs are almost unaffected by magnetic fields ${ }^{(8)}$. In contrast to the majority of APDs, SiPMs are very fast and have high gain. SiPMs are also of great interest since they enable determination of the Time of Flight (TOF) of the $511 \mathrm{keV}$ positron-electron annihilation photons ${ }^{(9)}$. The proper use of SiPMs, meaning a satisfactory discrimination of signal time, could result in a time resolution better than $100 \mathrm{ps}^{(10)}$. SiPMs feature a dark count rate (DC) that linearly increases with their active area size and could degrade the impinging photon position determination when using multiplexing read-out approaches ${ }^{(11)}$.

In this work we propose two approaches in order to use monolithic scintillator crystals for PET gamma detectors, based on PMTs and SiPMs, respectively. In the case of PSPMTs we will show the optimization of the light collection when reducing the AA by means of FPs. When working with SiPMs, in order to minimize the DC contribution, we have designed an array of SiPMs with a $1 \mathrm{~mm}^{2}$ detection area. Thus, to satisfy both requirements of small AA and small individual detection area, we have also proposed to use light guides to efficiently transfer the scintillation light to each SiPM (see Fig. 1).

\section{Materials and Methods}

We have modeled the effect of the scintillation light when transferred from the crystal to a photosensor array (PSPMT or SiPMs array) as a function of the AA. This work was carried out using Visual Basic programing language. A comparative study between simulated and experimental data using 
PSPMT has been performed. We have also simulated the results when an array of smaller active area SiPMs are used.

\subsection{Simulation data}

The modeled scintillation crystal has a truncated pyramidal shape of $48 \times 48 \mathrm{~mm}^{2}$ and $40 \times 40 \mathrm{~mm}^{2}$ for the large and small faces, respectively, and $12 \mathrm{~mm}$ thickness. We have simulated the behavior of the scintillation photons produced by incident $511 \mathrm{keV} \gamma$-rays. We calculated the photon trajectories including their impact position on the photosensor planar region.

Given the acceptance solid angle, the isotropical photon emission and the energy conservation, it is possible to determine the number of photons that will be emitted under such a solid angle as a consequence of the photon impact. The type of crystal we are considering is the so-called LYSO (LutetiumYttrium Oxyorthosilicate) which emits about 32000 photons/MeV at $420 \mathrm{~nm}$ wavelength. The refraction index $n_{L Y S O}$ is equal to 1.82 . The simulation also took into account that the quantum efficiency of the PSPMT for such light is $25 \%$. The $n$ for the borosilicate window at the entrance face of the PSPMT is $n_{P M T}=1.49$. The photons behave according to the Snell law when they change from one media to another characterized by the different refractive indexes.

In our design we avoid internal reflections on the sides of the crystal by black painting them thus preserving the angular distribution of the scintillation photons ${ }^{(5,6)}$. Therefore, the simulated photons whose rays impact such faces are, to a first approximation, not taken into account for the center of mass determination of the light distribution. We are aware that this is a simplified approach to the scintillation light behavior. A more accurate 
technique would require including effects like possible reflections of these photons due to the non total absorption of the paint or defects in the crystal face polishing.

\subsubsection{Monolithic crystals coupled to PSPMTs}

The simulated detection area is defined by $8 \times 8$ photodetectors (anodes) with individual dimensions of $6 \times 6 \mathrm{~mm}^{2}$ each, called PADs. In particular, we modeled the PSPMT H8500 from the company Hamamatsu ${ }^{(12)}$.

We performed simulations by considering several crystal-photosensor interfaces with different AAs. We modeled a layer of $150 \mu \mathrm{m}$ of optical grease with $n \approx 1.5$ and the use of FPs which accurately define the AA by their numerical aperture. The FPs are blocks composed of thousands of small size optical fibers which are stacked together capable to transmit light from the entrance to the exit faces ${ }^{(7)}$. Among other properties, the numerical aperture (NA) and the extra mural absorption (EMA) characterize these devices. The NA determines the maximum AA for a given refraction index of the fiber. In some cases, the FPs incorporate an attenuation material (EMA) which absorbs the light that does not enter the fiber and hits the fiber cladding or the light that escapes from the fiber.

Since the crystal performs similarly in all the regions, we have only modeled one eighth of the crystal with a total of 15 emission locations in the $X Y$ plane separated by $5 \mathrm{~mm}$ at different crystal depths, detached $1 \mathrm{~mm}$ (see Fig. 2). In every of these positions, we considered 100 photons $(511 \mathrm{keV})$. The $X Y$ positions are reconstructed by weighting the PAD signals: 

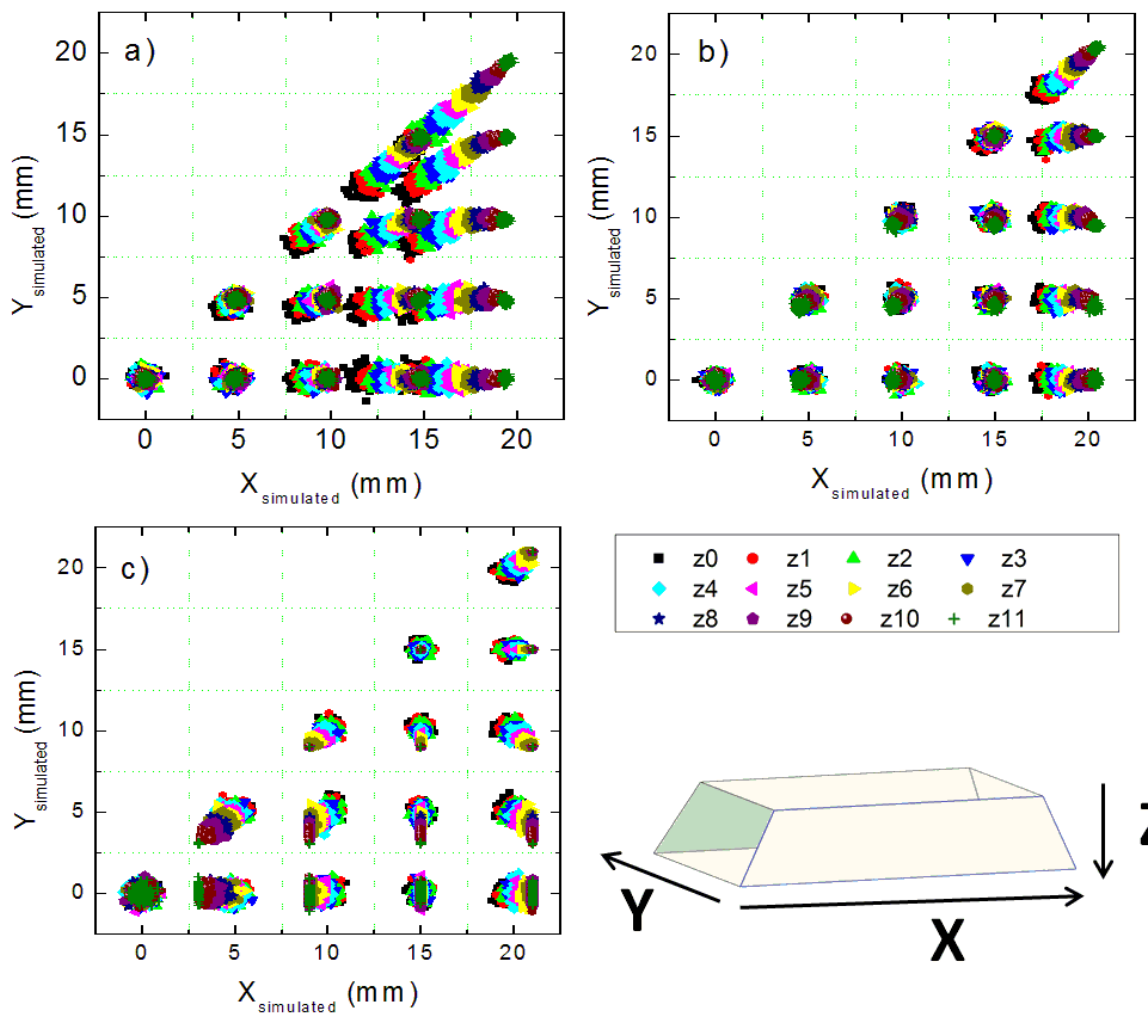

- $z 0 \bullet z 1 \quad z 2 \quad \nabla z 3$

- $44 \quad z 5>z 6 \quad z 7$

$\star z 8 \cdot z 9 \cdot z 10+z 11$

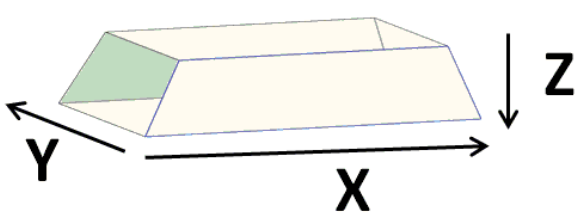

Figure 2: Simulation data of the $X Y$ photon impact determination of $\gamma$-ray sources. 15 positions located at one eighth of the crystal (12 mm thick) and separated by $5 \mathrm{~mm}$ each have been considered. The data was independently collected at different crystal depths $\left(Z_{i}, i=0 \ldots 11\right)$ in steps of $1 \mathrm{~mm}$, represented by the distinct colors and shapes in the plots. In each of these positions, we considered 100 photons $(511 \mathrm{keV})$. (a) Plot of results when using optical grease as coupling media between the crystal and the photosensor. (b) Same as before but using a faceplate with $\mathrm{AA}=33.3^{\circ}$ as coupling media. (c) Same as before but using a faceplate with $\mathrm{AA}=18.6^{\circ}$ as coupling media. (d) Sketch of the LYSO crystal and the axis directions.

$$
X=\frac{\sum_{i=1}^{8} x_{i} \cdot n_{i}}{\sum_{i=1}^{8} n_{i}}, \quad Y=\frac{\sum_{i=1}^{8} y_{i} \cdot n_{i}}{\sum_{i=1}^{8} n_{i}},
$$

where $n_{i}, x_{i}$ and $y_{i}$ are the number of collected scintillation photons, the 
$X$ and $Y$ coordinate center positions for the $i$ PAD, respectively. Figure 2 depicts the reconstructed $X Y$ positions as a function of different $Z$ photon impacts. Three coupling cases namely, optical grease $\left(\mathrm{AA}_{g} \approx 55^{\circ}\right)$, a faceplate with $\mathrm{AA}=33.3^{\circ}$ and a faceplate with $\mathrm{AA}=18.6^{\circ}$ respect to the normal, have been considered in this example. The maps depicted in Fig. 2 clearly show that the border effect is reduced when decreasing the AA, being almost negligible for the case of the $\mathrm{FP}$ with $\mathrm{AA}=18.6^{\circ}$. However, due to the strong constraint on the AA, when the event takes place very close to the exit face of the scintillator (the largest face with dimensions of $50 \times 50 \mathrm{~mm}^{2}$ ), the generated scintillation photons only reach one PAD and possibly its neighbors bringing on the consequent "PAD effect". The PAD centers are located at $X$ and $Y$ equal to $3,9,15$ and $21 \mathrm{~mm}$.

Figure 3 shows the resulting projections for the row $Y=0$ when considering optical grease and 3 distinct FPs $\left(\mathrm{AAs}=8.8^{\circ}, 18.6^{\circ}\right.$ and $33.3^{\circ}$ ) as coupling. These profiles also include the gamma ray penetration probability within the crystal. For $\mathrm{AA}=8.8^{\circ}$, is easily distinguishable that almost all events are assigned to a unique coordinate corresponding to the center PAD. The other three examples correspond to the projections of Fig. 2. In order to avoid scintillation light impinging only on one PAD, FPs with very small AA $\left(<10^{\circ}\right)$ were not considered further since they will cause very poor spatial resolution, especially for $511 \mathrm{keV}$ photon impacts near the exit face of the crystal block.

Instead of a point-like gamma-ray source we simulate the lateral distribution of a Gaussian function with a $\sigma=1.25 \mathrm{~mm}$ because we compare our simulations with experimental data obtained with a collimated source having 


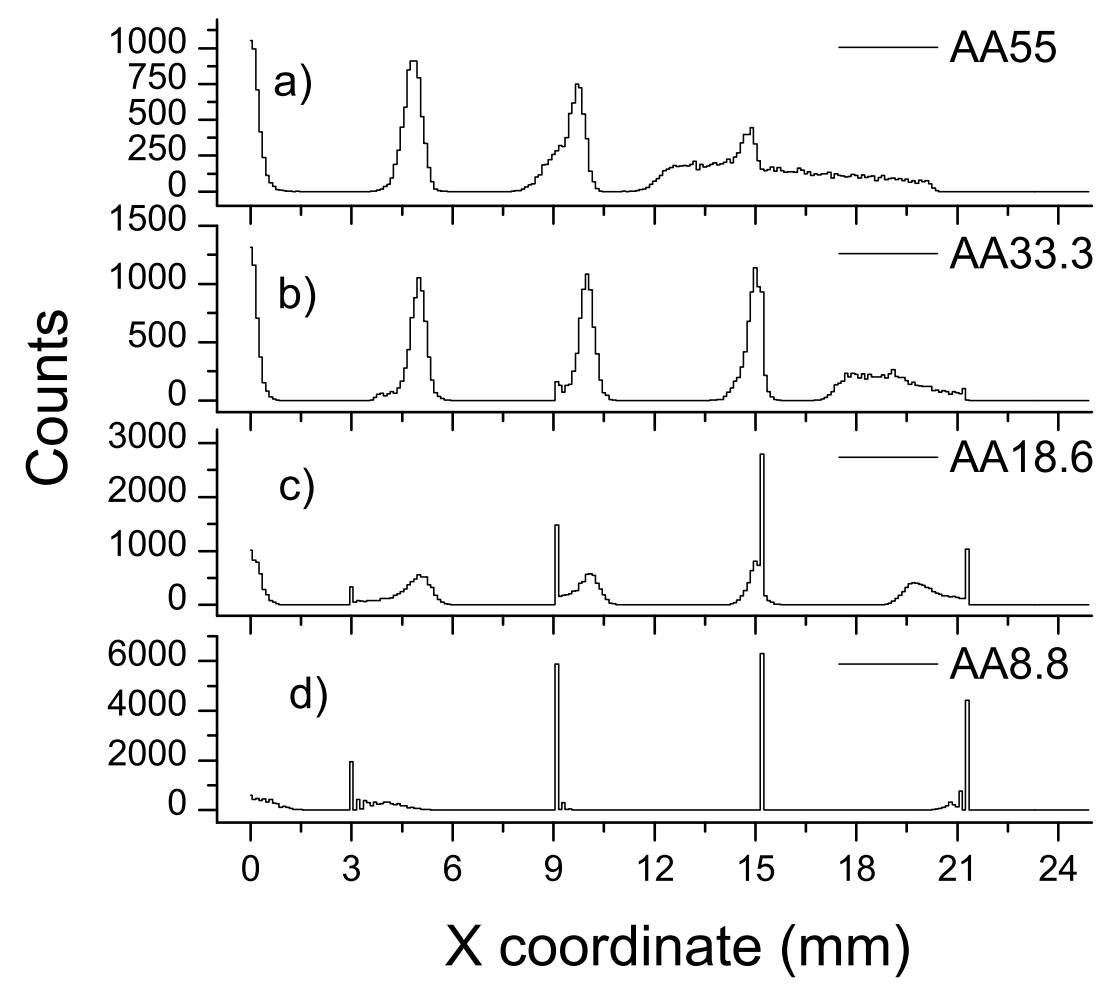

Figure 3: Simulation data for $Y=0$ including the summed results for $Z=0$ to 11 as a function of different AAs. a), b) and c) are projections of the data shown in Fig. 2. d) represent the projection for $\mathrm{AA}=8.8^{\circ}$ showing the PAD effect.

similar lateral characteristics. As will be detailed below, a collimator made out of Tungsten with drilled holes of $1.2 \mathrm{~mm}$ in diameter was used during the experiments, ensuring a perpendicular incidence of the $\gamma$-rays to the crystal entrance face. This degree of freedom also relates to a possible spread of the $511 \mathrm{keV}$ photons when they exit the collimator and before impacting the crystal surface. The refined modeling eliminated the spikes for cases in which a small AA is simulated (see Fig. 4). 
In addition to a finite lateral distribution of the emitted $\gamma$-rays, we also allowed for a fraction of the scintillation photons (app. 25\%) to internally reflect off the sides of the crystal. Figure 4 shows the results of these simulations for a sequence of different crystal thicknesses all coupled by means of optical grease to the H8500 PSPMT. These data include both the Gaussian spread of $\gamma$-ray photons at the crystal entrance and reflections of scintillation photons off the side walls.

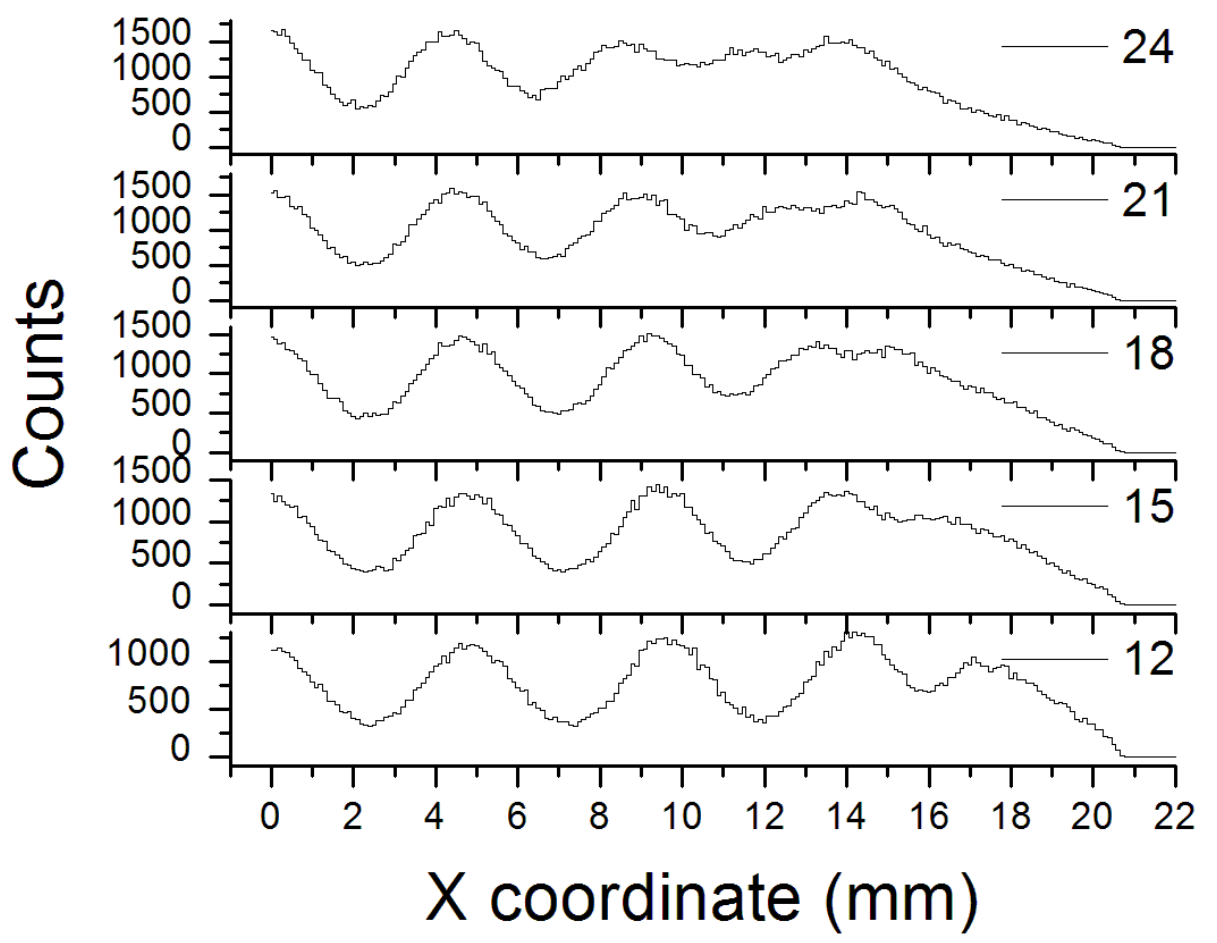

Figure 4: Simulation data for $Y=0$ including a Gaussian distribution of gamma rays and internal crystal reflections, using optical grease $\left(\mathrm{AA}_{g} \approx 55^{\circ}\right)$ as coupling media between crystal and PSPMT. The LYSO thicknesses vary from 24 (top) to $12 \mathrm{~mm}$ (bottom). 


\subsubsection{Monolithic crystals coupled to SiPMs}

We also modeled the distribution of scintillation light when the crystal is coupled to an array of SiPMs by means of optical light guides. These guides are optical concentrators which, funnel the light from a larger entrance area into a smaller output area at the expense of a reduced acceptance angle. They work as total internal reflection (TIR) devices. They are not metalized since they are more efficient when working as $\operatorname{TIR}^{(13)}$. The AA generated by these devices is app. $16^{\circ}$. The selected SiPM devices have an active area of $1 \times 1 \mathrm{~mm}^{2}$. The light concentrators also have an exit squared section of $1 \times 1 \mathrm{~mm}^{2}$ while the entrance one is of $3 \times 3 \mathrm{~mm}^{2}$. Thus, we considered in the new model analogue photosensor PADs of $3 \times 3 \mathrm{~mm}^{2}$ whose centers along a line were at $1.5,4.5,7.5,10.5,13.5,16.5,19.5$ and $22.5 \mathrm{~mm}$, respectively.

In Fig. 5 we have plotted the simulation results for a variety of crystal thicknesses when they are coupled to SiPMs by means of optical concentrators. These data do not include the $\gamma$-rays spread and internal photon reflections. We observe that the edge effect is considerably minimized even for crystals as thick as $24 \mathrm{~mm}$. For a crystal thickness lower than $15 \mathrm{~mm}$, since the geometrical lateral angle is also smaller than the AA of the light guidance elements, there is almost a negligible border effect. Nevertheless, we found some data concentrations at the PAD centers even for the $21 \mathrm{~mm}$ thick crystal. It must be noted that this effect is slightly higher than the one observed for a crystal of $15 \mathrm{~mm}$ thickness and photosensors of $6 \times 6 \mathrm{~mm}^{2}$ (PSPMT case). 


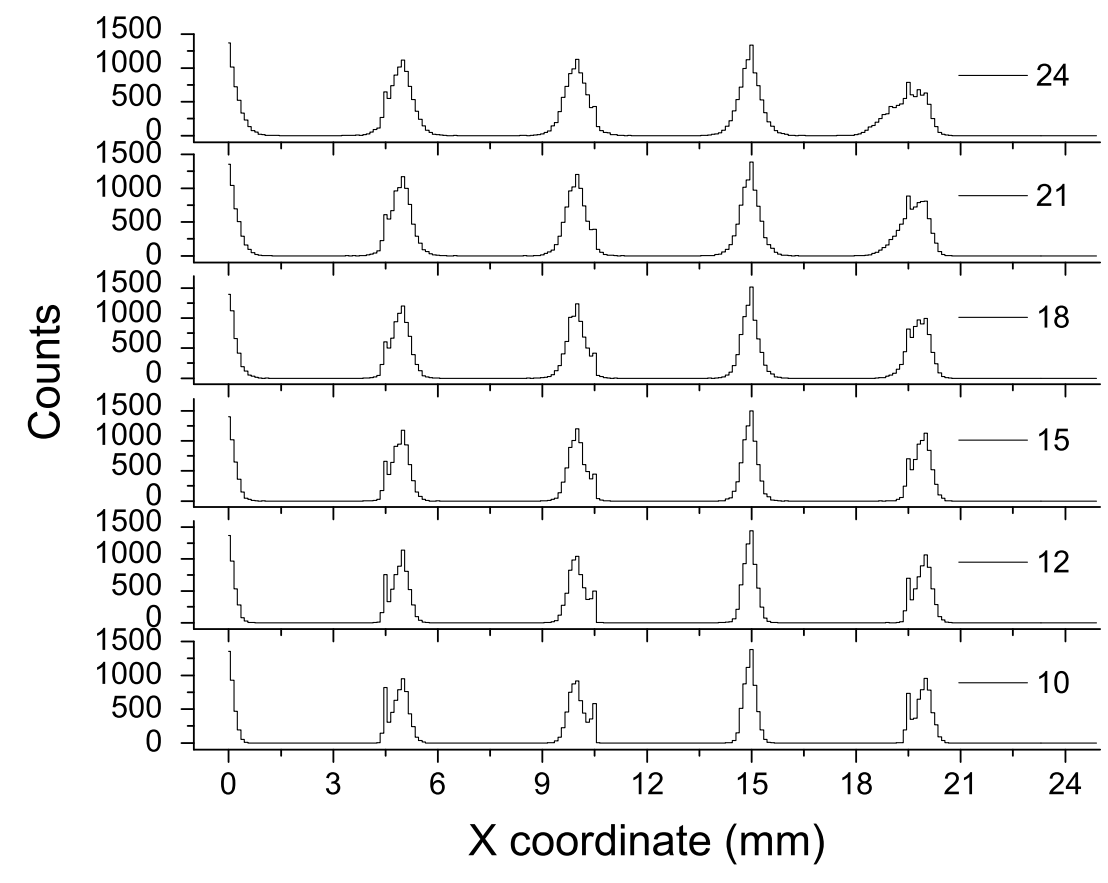

Figure 5: Simulation data for $Y=0$ for different crystal varying from 10 (bottom profile) to $24 \mathrm{~mm}$ thicknesses (top profile) coupled to an array of SiPMs by optical concentrators.

\subsection{Experimental data}

The experimental data was taken using a variety of different scintillation LYSO crystals with thicknesses ranging from 10 to $24 \mathrm{~mm}$. An array of well known positioned $81{ }^{22} \mathrm{Na}$ sources was used to study light position and energy. The center-to-center source distance was $5 \mathrm{~mm}$. The total activity of the array was about $5 \mu \mathrm{Ci}$. The $1 \mathrm{~mm}^{2}$ extended sources were collimated by means of the Tungsten block (24 mm thick) with drilled holes of $1.2 \mathrm{~mm}$ in diameter. 


\subsubsection{Acquisitions based on PSPMTs}

Two opposite detector blocks formed by a PSPMT H8500 from Hamamatsu. The signals of the two detector blocks were fed into a DAQ system composed of a Trigger and two Analogue to Digital Converters (ADC) modules. The last dynode signal of every PSPMT was injected into the Trigger requiring a coincidence within a 5 ns time window. These coincidences triggered the digitalization of 4 multiplexed signals enabling the $X$ and $Y$ location of the photon impact coordinate ${ }^{(3,7)}$. As in the case of the simulation, different coupling media were used to test the edge effect and energy resolution of the system.

The effect of image compression due to an increase of the crystal thickness was already reported in a previous study ${ }^{(7)}$. In the current work we perform an exhaustive analysis of both the energy resolution and the compression effect, as a function of different AAs. As an example of this effect, a sequence of images for two different crystal thicknesses using three distinct AAs is shown in Fig. 6. Every image depicts the central row of the $\mathrm{Na}$ array containing, therefore, 9 sources. The profiles were fitted to a multi-Gaussian distribution. The angular acceptance was $33.3^{\circ}$ (FP47A or air), $18.6^{\circ}$ (FP75C) and $54.6^{\circ}$ (Grease). Note that FP47A and FP75C are the faceplate codes used by the provider Schott ${ }^{(14)}$. In this figure we observe that the border effect reduces when decreasing the AA, although it does not properly work for the FP75C. As we stated in a previous work ${ }^{(7)}$, this is caused by the fact that the FP75C does not incorporate EMA and, therefore, a certain amount of light passes through the optical fibers increasing the AA from $18.6^{\circ}$ to something around $45 \pm 5^{\circ}$. 

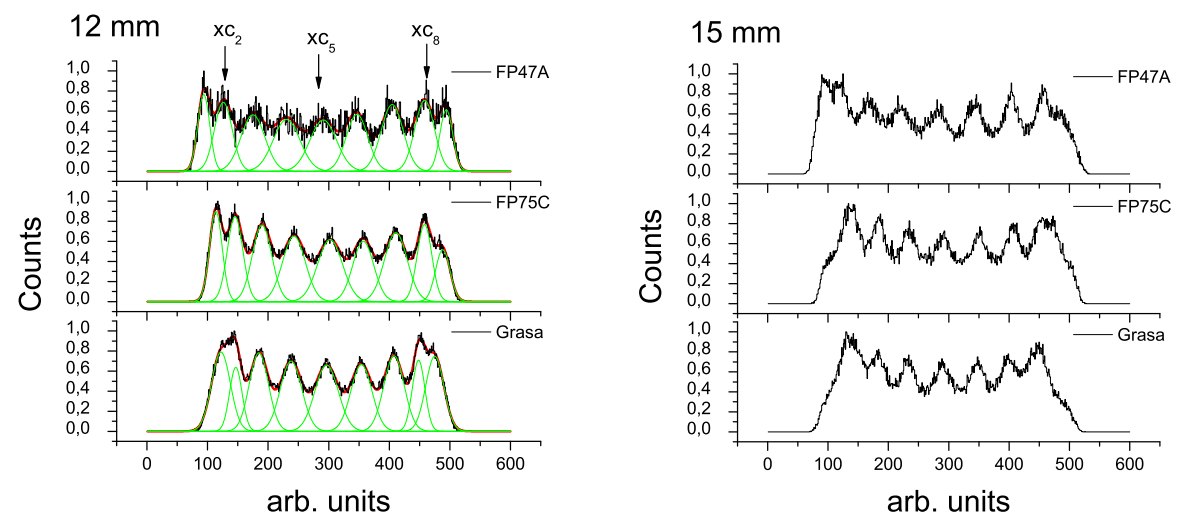

Figure 6: Examples of $9{ }^{22} \mathrm{Na}$ sources equally spaced by $5 \mathrm{~mm}$ for two crystal thicknesses (12 $\mathrm{mm}$ and $15 \mathrm{~mm}$, left and right, respectively) and as a function of different coupling media. The AA for Grease and FP47A is $54.6^{\circ}$ and $33.3^{\circ}$, respectively. Concerning the AA for the FP75C see the text.

In order to evaluate the degree of compression caused by the different coupling media and the crystal thickness, we have plotted the difference between separated centers of the measured profiles. In particular, as shown in Fig. 7, we have depicted the difference between the two next to last peaks (labeled as $\mathrm{xc}_{2}$ and $\mathrm{xc}_{8}$ in Fig. 6, respectively) with respect to the central one denoted as $\mathrm{xc}_{5}$.

The plots depicted in Fig. 7 show that the distance from the $\mathrm{xc}_{5}$ to the $\mathrm{xc}_{2}$ and $\mathrm{xc}_{8}$ centroids increases with the $\mathrm{AA}$, as it was expected. We can observe that the edge effect generates a strong compression of the peaks for thick crystals when using optical grease as coupling media. In these cases, we notice that only below $15 \mathrm{~mm}$ thickness is there an almost linear dependency. For the case of the FP47A $\left(33.3^{\circ}\right)$, we can see a longer linear behavior up to 


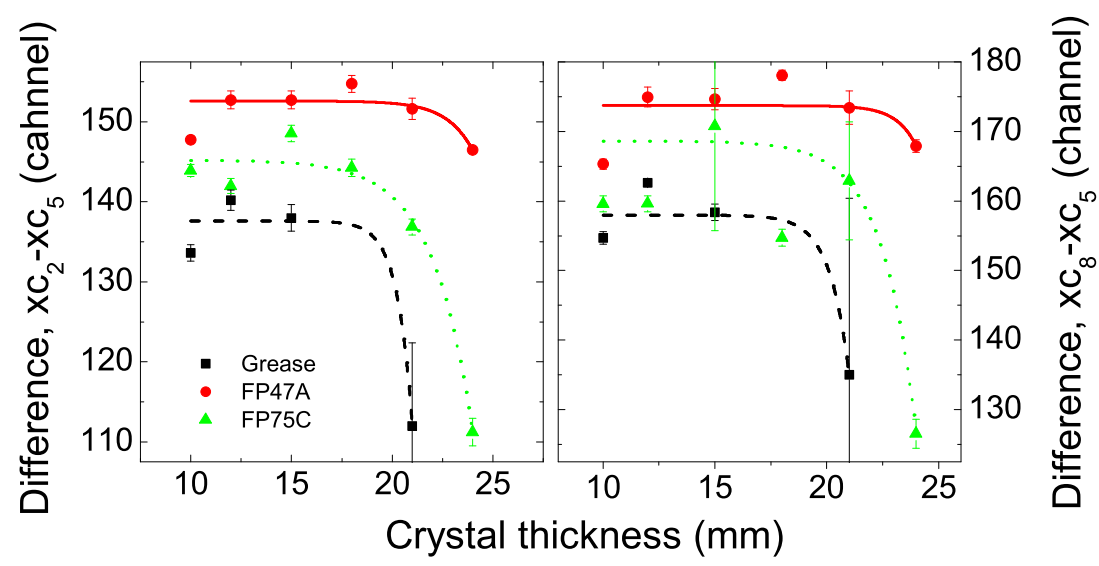

Figure 7: Image compression effect measured through peak differences as a function of the crystal thickness and AA.

at least $18 \mathrm{~mm}$ thickness.

We also studied the energy resolution of the detector block as a function of different crystal thicknesses and AAs. For these measurements we considered a region of interest around the central ${ }^{22} \mathrm{Na}$ source of the array. Such a small area reduces the contributions of several PADs with different gains to the final energy resolution. During the analysis we observed the $511 \mathrm{keV}$ annihilation peak but also the $1.274 \mathrm{MeV}$ gamma ray characteristic of the ${ }^{22} \mathrm{Na}$. The linearity of the system was determined and validated by determining the ratio of both peak positions at $2.52 \pm 0.09$ (theoretical value 2.49 ). We fitted the $511 \mathrm{keV}$ photopeak to a custom function defined by a Gaussian distribution on top of a tilted background. The energy resolution is given by the ratio of the FWHM to the energy centroid.

In Fig. 8 left we have plotted the energy resolution as a function of the 

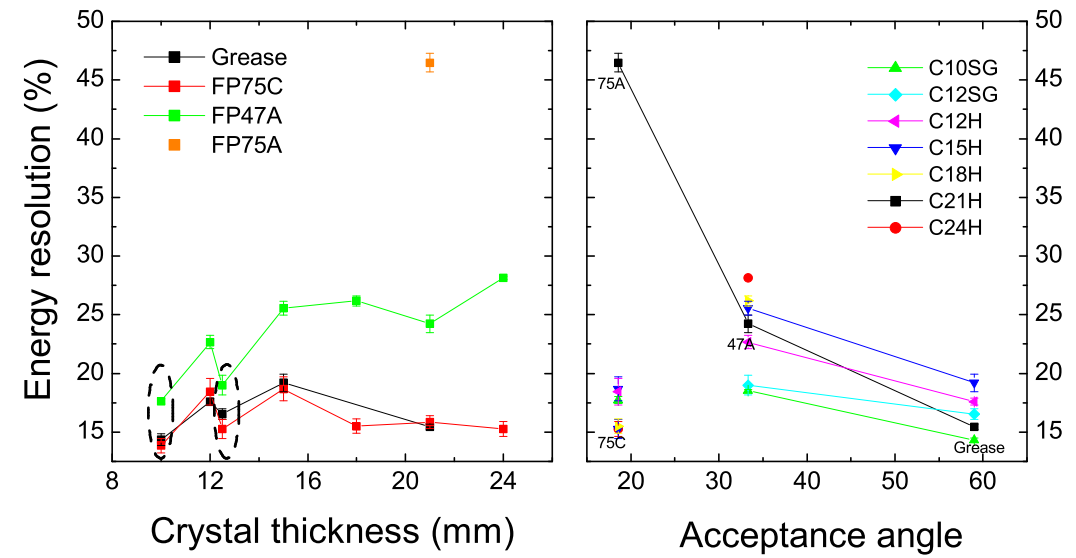

Figure 8: Energy resolution as a function of the crystal thickness (left) and of the AA (right). Note that the label $75 \mathrm{~A}$ refers to the $\mathrm{FP}$ with $\mathrm{AA} \approx 18.6^{\circ}$, see Fig. 2 . The data surrounded in the left figure with a dashed-line ellipsoid was acquired with crystals supplied by Saint Gobain, others were supplied by Hilger Crystals. The legend on the right graph differentiates for crystals supplied by Saint Gobain (SG) or Hilger Crystals (H). Since the exact AA for the data collected with the FP75C is unknown (see text for details), they are not connected by lines with the results obtained with the other configurations.

crystal thickness and for the various coupling media. We could state that the energy resolution shows no dependency with the crystal thickness but on AA, as expected. The difference between the results for the FP75C and FP75A are a consequence of the above mentioned missing EMA on the FP75C. Fig. 8 right shows the results for the energy resolution as a function of the different coupling media (AA) and for a variety of the crystal thicknesses. We observe that the lower the AA is, the worse the energy resolution becomes. This occurs since the amount of transferred light reduces along with the AA, directly translating into a poor energy resolution. Although the AA for the 
results obtained with the $\mathrm{FP} 75 \mathrm{C}$ have been assigned to an $\mathrm{AA}=18.6^{\circ}$, the experiment confirms that the missing EMA produces an effective AA larger than $18.6^{\circ}$

\subsubsection{SiPM data}

The results previously described were acquired with sensor blocks containing a PSPMT as photosensor and using FPs or optical grease as coupling media between the crystal and the photosensor. Preliminary experimental data was obtained when using an array of light guides mounted in between the scintillator and an assembly of SiPMs. The optical manufacturing design of the light guide was optimized using the ZEMAX program that is based on light propagation within the crystal and guides. An optimal configuration that represents a balance between light detection efficiency (70\%) and crosstalk (12 dB) between channels was found. This configuration has already been implemented by developing a special cast for an array of 8 light guides using PMMA as material. A final matrix of 256 light guides is built by gluing 32 of those arrays together with the help of a plastic grid (see Fig. 1c). An array of 16x16 SiPMs was mounted to the back of a scintillator interfacing 256 light guides in between. The crystal has dimensions of $40 \times 40 \mathrm{~mm}^{2}$ and $50 \times 50 \mathrm{~mm}^{2}$ for the entrance and exit faces, respectively, and a thickness of $12 \mathrm{~mm}$. We use four Application Specific Integrated Circuits (ASIC) capable of reading $64 \mathrm{SiPMs}$ each ${ }^{(15)}$. Since all SiPMs were connected to the same power supply, they exhibit a significant gain difference among them which was compensated for by the ASIC programming ${ }^{(16)}$. 


\section{Comparison and discussion}

We first compared the simulated and real data using the PSPMT as photosensor. In terms of image compression and spatial resolution, Fig. 9 shows a comparative projection between the experimentally measured and the simulated data, for a crystal of $10 \mathrm{~mm}$ thickness coupled by means of optical grease. As previously described, these profiles depict a row of nine ${ }^{22} \mathrm{Na}$ sources. The simulation, which included the $\gamma$-rays spread and the internal reflection tends to better resolve the edge sources. These data also show a better signal to background ratio.

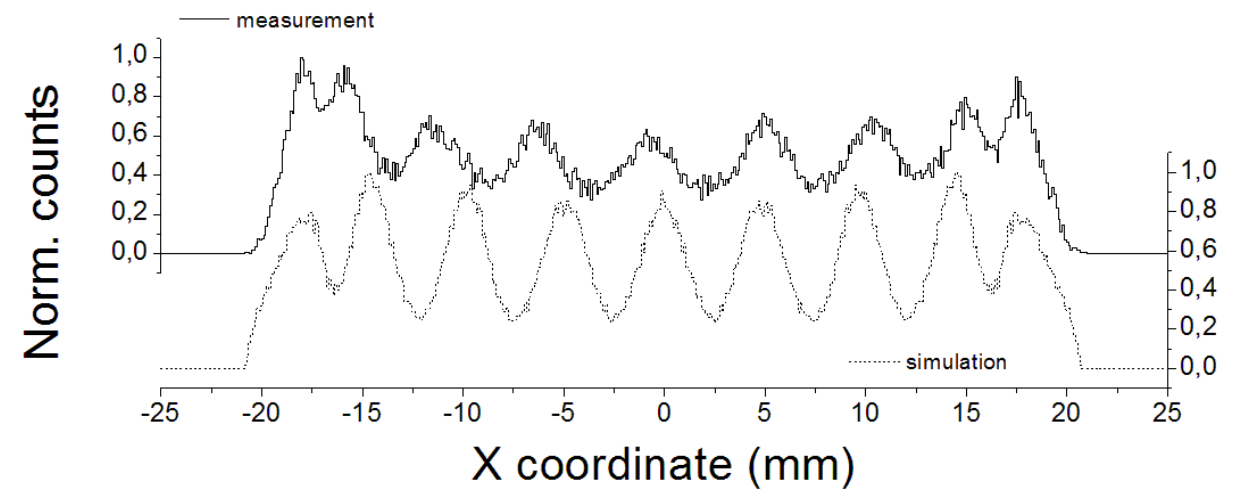

Figure 9: Comparison between experimental and simulated data for an array of nine ${ }^{22} \mathrm{Na}$ sources, using optical grease to couple the scintillator crystal and the PSPMT. The crystal thickness was $10 \mathrm{~mm}$.

When comparing the data using SiPMs as photosensors, there is a lower correlation between experimental and simulated data. Figure 10 shows on the top, experimental profiles acquired with the 256 SiPMs array and light guides and, on the bottom, the simulated data under similar conditions. The modeled data included, as in the case of the PSPMTs, a $\gamma$-ray spread of 
$\sigma=1.25$ and a percentage of reflections in the absorbent faces. In these tests we used single ${ }^{22} \mathrm{Na}$ sources (1 $\mathrm{mm}$ in diameter) moved in steps of $5 \mathrm{~mm}$. We observe a quite low spatial resolution of the experimental data when compared to the simulation. A compression effect of the experimental data is observed. This could be explained if we consider that the theoretical AA value of $16^{\circ}$ was not achieved in our experimental set-up, being larger than this. However, independently of the percentage of reflections considered, we have not observed this effect in our simulations. Since the model did not take into account pure SiPM effects such as crosstalk between SiPMs or the read out electronics, this could have caused the discrepancy between the experimental and the modeled data.

The energy resolution obtained with this set-up has a value of $41 \pm 2 \%$ at the $511 \mathrm{keV}$ photopeak. Indeed, following the curve obtained in Fig. 8 left, the current result matches well with that distribution considering that the AA for the light guides is $16^{\circ}$.

The experimental comparison between the case of using SiPMs with an $\mathrm{AA}=16^{\circ}$ (see Fig. 10 top) and a PSPMT (see Fig. 6 left) suggests that due to the reduced AA of the light guides, there is poor collection of light by the SiPMs array. Therefore, both the energy and spatial resolutions are strongly affected. This AA implies that for a crystal thickness of $12 \mathrm{~mm}$, the generated 2D light cone for $\gamma$-rays interacting just on the entrance face will cover only approximately $7 \mathrm{~mm}$. Moreover, the $511 \mathrm{keV}$ rays impinging normally to the entrance face will, on average, interact at about $5 \mathrm{~mm}$ depth ${ }^{(17)}$. For this reason in most cases only one single light guide is illuminated, and therefore, only a single SiPM. This results in the poor spatial resolution measured for 


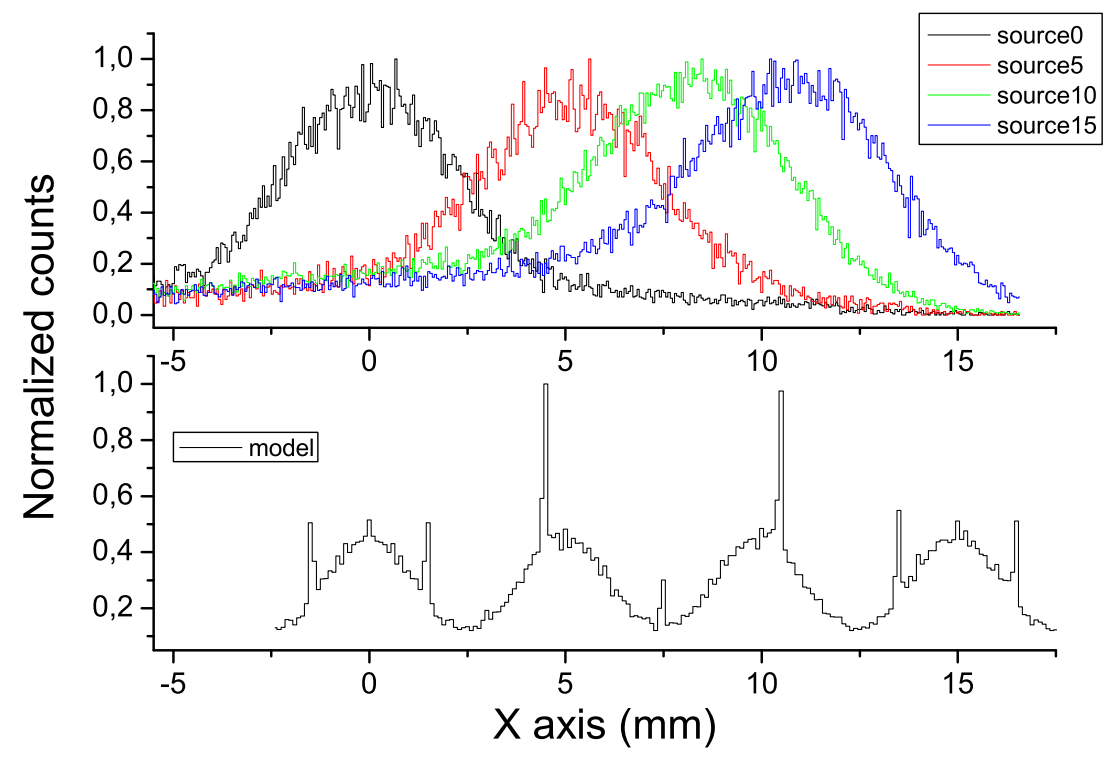

Figure 10: Comparison between experimental (top) and simulated (bottom) data for five ${ }^{22} \mathrm{Na}$ sources, using the light guides as coupling media between the scintillator and an array of SiPM. The crystal thickness was $12 \mathrm{~mm}$.

this crystal thickness.

\section{Conclusions}

In this paper we have shown the possibility of modeling the scintillation light within a crystal and its transfer to the photosensor applying acceptance angular restrictions. SiPM detectors are currently being studied in order to replace PMT technology due to its performance, especially related to time resolution and magnetic field compatibility. The presented data has been compared with experimental measures under similar and controlled conditions. Both simulation and experimental data were obtained for a large 
variety of crystal thicknesses and different angular constraints. It is possible to avoid the border effect by reducing the AA as was demonstrated. However, when a very small AA is selected, most of the light is collected only by a single photosensor which increases the PAD effect. A balance between border and PAD effects must be found.

The study can be divided in two groups; the data simulated and acquired for a PSPMT as photosensor and those obtained when an array of SiPMs is used. In general, there is a close correlation between the simulated and measured data when considering the PSPMT in the sensor block, especially when internal reflections within the crystal are considered. This study clearly showed that the collected statistics strongly depended on AA reduction, worsening both spatial and energy resolutions, as was expected. In case of using PSPMT as a photosensor, we have demonstrated that it is possible to increase the crystal thickness of the detector block without increasing the border effect. This can be achieved using a FP as a coupling device between the scintillator and the PSPMT with an AA of about $33^{\circ}$, without a significant detriment to the energy resolution.

When the array of SiPMs with reduced effective detection area was considered, the data correlated well with the expected energy resolution (see Figs. 10c and 8), but poorly in terms of spatial resolution. Nevertheless, we expect that even for a thick crystal of about $24 \mathrm{~mm}$, the number of impacted SiPMs could not be enough to obtain satisfactory spatial resolution. To solve this behavior, we intend to reduce the concentration power of the light guides, meaning the use of a smaller entrance area. This will permit the increase of the AA angle but also the number of illuminated SiPMs. 
In contrast to these expected improvements, we will also increase the DC rates. Here, current manufacturing technologies have considerably improved to reduce the total DC rates.

\section{Acknowledgement}

This work was supported by the Centre for Industrial Technological Development co-funded by FEDER through the Technology Fund (DREAM Project, IDI-20110718), the Spanish Plan Nacional de Investigación Científica, Desarrollo e Innovación Tecnológica (I+D+I) under Grant No. FIS201021216-CO2-01 and the Valencian Local Government under Grant PROMETEO 2008/114.

[1] M.S. Judenhofer et al., Simultaneous PET-MRI: a new approach for functional and morphological imaging, Nature Medicine 14 (2008) 459465.

[2] A. Drzezga, M. Souvatzoglou, M. Eiber, A.J. Beer, S Fürst, A. Martinez-Möller, S.G. Nekolla, S. Ziegler, C. Ganter, E.J. Rummeny and M. Schwaiger, First Clinical Experience with Integrated WholeBody PET/MR: Comparison to PET/CT in Patients with Oncologic Diagnoses, J. Nucl. Med. 53 (2012) 845-855.

[3] J.M. Benlloch, V. Carrilero, A.J. González, J. Catret, C.W. Lerche, D. Abellán, F. García de Quirós, M. Martínez, J. Modia, F. Sánchez, N. Pavón, A. Ros, J. Martínez and A. Sebastiá, Scanner calibration of a small animal PET camera based on continuous LSO crystals and flat panel PSPMTs, Nucl. Instr. and Meth. A 571 (2007) 26-29. 
[4] D.R. Schaart, H.T. van Dam, S. Seifert, R. Vinke, P. Dendooeven, H. Löhner and F.J. Beekman, A novel, SiPM-array-based, monolithic scintillator detector for PET, Phys. Med. Biol. 54 (2009) 3501-3512.

[5] F. Sánchez, L. Moliner, C. Correcher, A. González, A. Orero, M. Carles, A. Soriano, M.J. Rodríguez-Alvarez, L.A. Medine, F. Mora and J.M. Benlloch, Small animal PET Scanner based on monolithic LYSO crystals: performance evaluation, Med. Phys. 39(2) (2012) 643-563.

[6] C.W. Lerche, A. Ros, V. Herrero, R. Esteve, J.M. Monz, A. Sebasti, F. Snchez, A. Munar and J.M. Benlloch, Dependency of Energy-, Positionand Depth of Interaction Resolution on Scintillation Crystal Coating and Geometry, IEEE Trans. Nucl. Sci. 55 (2008) 1344-1351.

[7] A.J. González Martínez, A. Peiró Cloquell, F. Sánchez Martínez, L.F. Vidal San Sebastian and J.M. Benlloch Baviera, Innovative PET detector concept based on SiPMs and continuous crystals, Nucl. Instrum. Meth. A 695 (2012) 213-217.

[8] S. España, L.M. Fraile, J.L. Herraiz, J.M. Udías, M. Desco and J.J. Vaquero, Performance evaluation of SiPM photodetectors for PET imaging in the presence of magnetic fields, Nucl. Instrum. Meth. A 613 (2010) 308-316.

[9] C.L. Kim, G. Wang and S. Dolinsky, Multi-Pixel Photon Counters for TOF PET Detector and Its Challenges, IEEE Trans. Nucl. Sci. 56 (2009) $2580-2585$. 
10] J. Torres, A. Aguilar, R. García-Olcina, J. Martos, J. Soret, J.M. Benlloch, A.J. González and F. Sánchez, High resolution Time of Flight determination based on reconfigurable logic devices for future PET/MR systems, Nucl. Instrum. Meth. A 702 (2013) 73-76.

[11] P. Dokhale, C. Stapels, J. Christian, Y. Yang, S. Cherry, W. Moses and K. Shah, Performance Measurements of a SSPM-LYSO-SSPM Detector Module For Small Animal Positron Emission Tomography, IEEE Nucl. Scien. Sympos. Conf. Record (2009) 2809-2812.

[12] www.hamamatsu.com.

[13] J.C. Chaves, Introduction to nonimaging optics, CRC Press, Taylor \& Francis Group, LLC (2008).

[14] www.schott.com.

[15] V. Herrero-Bosch, C.W. Lerche, M. Spaggiari, R. Aliaga-Varea, N. Ferrando-Jodar and R. Colom-Palero, AMIC: An Expandable FrontEnd for Gamma-Ray Detectors With Light Distribution Analysis Capabilities, IEEE Trans. Nucl. Sci. 58 (2011) 1641-1646.

[16] P. Conde, A. J. González, L. Hernández, L. Moliner, A. Orero, M. J. Rodríguez, F. Sánchez, A. Soriano, L. F. Vidal and J. M. Benlloch, First results of an ASIC controlled $\gamma$-detector based on a SiPM-array and a monolithic LYSO, IEEE 2012 NSS-MIC Conference Record (2013) 412414.

[17] A. Soriano, A.J. González, F. Sánchez, P. Conde, L.a Moliner, A. Orero, M.J. Rodríguez-Alvarez, L.F. Vidal and J.M. Benlloch, Minimization of 
parallax error in breast dedicated PET, IEEE Trans. Nucl. Sci. 60 (2013)

429 739-745. 